Short communication

\title{
A comparison of drug-eluting stent versus balloon angioplasty in patients with bare-metal stent instent restenosis: 5 year outcomes 2 s
}

\author{
Abdulmelik Yıldız ${ }^{\mathrm{a}}$, Cennet Yıldız ${ }^{\mathrm{b}, *}$, Vecih Oduncu ${ }^{\mathrm{a}}$, Arif Oğuzhan Çimen ${ }^{\mathrm{a}}$ \\ a Medical Park Hospital, Istanbul, Turkey \\ b Tekden Hospital, Istanbul, Turkey
}

\section{A R T I C L E I N F O}

\section{Article history:}

Received 16 December 2015

Received in revised form 4 February 2016

Accepted 4 February 2016

Available online 3 March 2016

\section{Keywords:}

Bare metal stent

Drug eluting stent

Coronary revascularization

Saphenous vein graft

\begin{abstract}
A B S T R A C T
Objectives: The objective of the present study was to compare the long term outcomes of balloon angioplasty (BA) versus drug-eluting stents (DES) in bare-metal stent in-stent restenosis (BMS-ISR).

Background: Coronary in-stent restenosis (ISR) remains a significant clinical problem. Long term results after management of ISR may help improve treatment strategies.

Methods: We assessed 5-year clinical outcomes in cohort of 269 patients with BMS-ISR treated with DES ( $\mathrm{n}=$ 154) and BA ( $\mathrm{n}=115)$ between June 2007 and January 2010 at our institution.

Results: Clinical and demographic characteristics were similar for both groups. Mehran classification was used to classify ISR lesions. BA were used predominantly in classes I and II, whereas classes III and IV were treated with DES ( $p<0.0001$ ). Percentages of major adverse cardiovascular events (MACE) including death, myocardial infarction (MI) and target vessel revascularization (TVR) for $4.37 \pm 1.1$ years were $50.4 \%$ and $31.8 \%$ for the BA and DES groups, respectively $(\mathrm{p}=0.002)$. Although patients in the BA group had significantly higher rates of recurrent angina ( $42.6 \%$ vs. $27.3 \%, p=0.009)$ and TVR (37.4\% vs. $20.8 \%, p=0.003)$, MI $(6.1 \%$ vs. $5.2 \%, p=0.752)$ and cardiac death (21.7\% vs. $16.2 \%, \mathrm{p}=0.251)$ were similar in both groups. MACE-free 1-year survival and 5-year survival rates were significantly higher in DES group compared to BA group (1 year survival: $91.6 \%$ vs. 71.3 $\mathrm{p}<0.001$, and 5 year survival: $68.2 \%$ vs. $49.6 \%, \mathrm{p}<0.0001$, respectively).

Conclusions: Although DES were more frequently used in to treat complicated lesions in patients with ISR, followup MACE rates were significantly lower and MACE-free survival was significantly better in the DES treated patients.

(C) 2016 The Society of Cardiovascular Academy. Production and hosting by Elsevier B.V. All rights reserved. This is an open access article under the CC BY-NC-ND license (http://creativecommons.org/licenses/by-nc-nd/4.0/).
\end{abstract}

\section{Introduction}

Development of bare metal stents (BMS) has become a major advancement in the treatment of coronary artery disease. BMS reduce restenosis rates by attenuating arterial recoil and contraction as compared to balloon angioplasty. However, in-stent restenosis (ISR) still occurs in approximately $10-20 \%$ of cases. ${ }^{1}$ Despite high rates of restenosis, BMS are widely used for treating coronary artery disease. ${ }^{2}$ Treatment of ISR remains a major challenge for clinicians. There are many treatment options for patients having ISR like recurrent balloon angioplasty (BA), drug-eluting stents (DES) or BMS, cutting balloon angioplasty, directional coronary atherectomy, rotational coronary atherectomy and vascular brachytherapy. ${ }^{3-6}$ Although vascular brachytherapy is an effective

\footnotetext{
4 Peer review under responsibility of The Society of Cardiovascular Academy.

* Corresponding author at: Konaklar mah. Org. İzzettin Aksalur cad. Oyak sitesi sok. 38, Blok Daire 2, Beșiktaș, İstanbul, Turkey. Tel.: + 90 5337469996; fax: + 902123698553.

E-mail addresses: drmelik@hotmail.com (A. Yıldız), cennet_yildiz@live.com (C. Yıldız), voduncu@yahoo.com (V. Oduncu), arif_cimen@yahoo.com (A.O. Çimen).

Peer review under responsibility of The Society of Cardiovascular Academy.
}

treatment of ISR, it requires additional personnel, training and equipment. BA may be preferred in patients with contraindications for dual antiplatelet therapy (DAPT). Weintraub et al. showed that restenosis that developed following successful BA has no adverse effect on longterm survival. $^{7}$

In this study, we compared the long term results of new-generaton DES with those of BA in patients presenting with BMS-ISR.

\section{Material and methods}

Patients

We analyzed clinical and angiographic data of patients who underwent PCI in our institution between June 2007 and January 2010. A total of 398 patients developed BMS-ISR during the study period. Of the 398 patients, 88 had DES-ISR, 11 patients underwent coronary artery bypass graft surgery, 9 patients underwent hybrid coronary revascularization, 3 patients refused percutanous intervention, and 18 patients were lost to follow-up. The remaining 269 patients who were treated with balloon angioplasy or DES enrolled in the study. Patients 


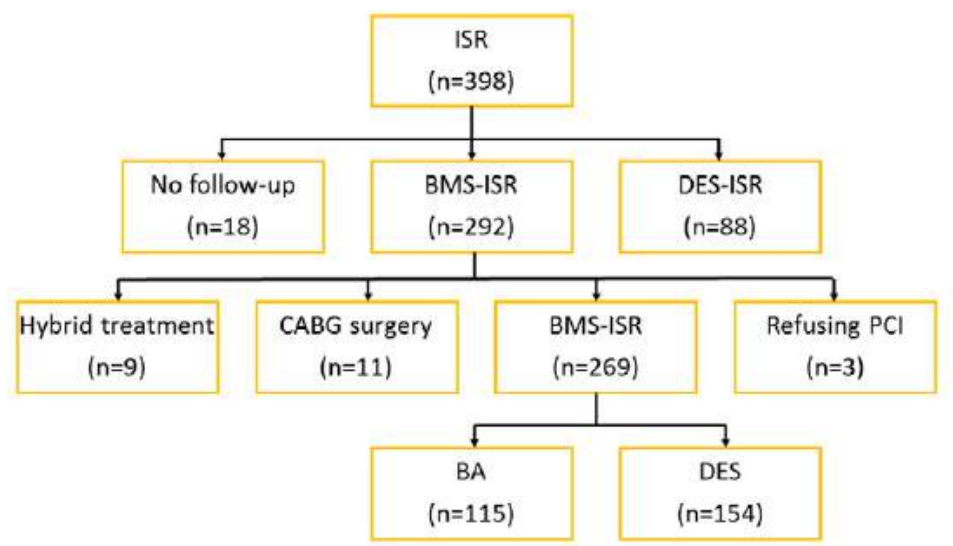

BA; balloon angioplasty, BMS-ISR; bare metal stent in-stent restenosis, CABG surgery; coronary artery bypass graft surgery, DES; drug eluting stent, DES-ISR; drug eluting stent in-stent restenosis, ISR; in-stent restenosis, PCl; percutaneous coronary intervention.

Fig. 1. Patient and treatment group profile. BMS ISR; bare metal in-stent restenosis, DES; drug eluting stent, DES ISR; drug eluting stent in-stent restenosis, ISR; instent restenosis.

were assigned to balloon angioplasty group ( 115 patients) or DES group (154 patients). Fig. 1 shows diagram of patients included and excluded in the study. Follow-up for all patients was continued until July 2013. The study was approved by the local ethics committee.

\section{Clinical and laboratory evaluation}

A medical history was taken from each patient, followed by a physical examination. Patient data were extracted from electronic medical records. The collected data included patient demographics, clinical characteristics, risk factors (arterial hypertension, diabetes mellitus, smoking, family history of coronary artery disease, dyslipidemia), medications, previous invasive cardiac procedures and echocardiographic findings including left ventricular ejection fraction (LVEF). Patients were excluded if they had active infection, anemia, renal failure, hepatic disease and thyroid function abnormalities. Patients received DAPT for four weeks after BMS implantation. Coronary angiography was performed to define coronary anatomy in patients who developed anginal symptoms, unstable angina, myocardial infarction (MI) and ischemic findings on nonivasive testing.

\section{Coronary intervention}

Coronary interventions were performed according to current practice guidelines and the results were recorded digitally for quantitative analysis. Degree of coronary stenosis was estimated visually by two experienced interventional cardiologists.

Definitions were based on predetermined criteria.

a- ISR was defined as $>50 \%$ narrowing of the lumen diameter according to the results of follow-up coronary angiographies.

b- The Mehran and American College of Cardiology/American Heart Association classifications were used to assess lesion shape. ${ }^{8}$ The classification is based on the length and pattern of the restenotic lesion in relation to the stented portion of the vessel. Four types of ISR have been defined: (I) focal ( $\leq 10 \mathrm{~mm}$ length); (II) diffuse (ISR $>10 \mathrm{~mm}$ within the stent); (III) proliferative (ISR $>10 \mathrm{~mm}$ extending outside the stent); and (IV) occlusive ISR.

c- Target vessel revascularization (TVR) was defined as repeat percutanous coronary intervention within the index procedure stent or $5 \mathrm{~mm}$ edge. ${ }^{9-10}$

d- All deaths were considered to be cardiac related unless a clear noncardiac cause could be established.

e- The diagnosis of MI required 2 of the following: 1) prolonged (>30 min) chest pain; 2) a rise in creatine kinase levels exceeding twice the local upper normal limit value (with abnormal MB fraction); and 3) development of persistent ischemic electrocardiographic changes (with or without new pathological Q waves). ${ }^{11}$

f- The Academic Research Consortium definition was used to assess the presence of stent thrombosis. ${ }^{12}$

g- Significant coronary stenosis was defined as 50\% narrowing of the lumen diameter in major epicardial coronary vessels. ${ }^{13}$

All patients received clopidogrel (300 to $600 \mathrm{mg}$ ) at least $6 \mathrm{~h}$ before the stent implantation. They also received weight-adjusted intravenous heparin before the intervention. Procedural success was defined as reduction of stenosis to less than $10 \%$ residual narrowing, with improvement in ischemic symptoms and without major procedure related complications: death, emergency bypass surgery, or myocardial infarction (defined to be greater than twice the increase in creatine kinase-MB levels). ${ }^{14}$

Drug eluting sirolimus stent or drug eluting paclitaxel stent was used instent restenosis. Balloon size was selected in order to achieve a final balloon-to-artery ratio of $1.1 / 1$. Relatively high pressures (>12 atm) were recommended.

The patients were premedicated with aspirin $100 \mathrm{mg} /$ day, and were given clopidogrel (loading dose of 300 to $600 \mathrm{mg}$ ) at least $6 \mathrm{~h}$ before the intervention. The patients were advised to stay on clopidogrel for one year after stent implantation. All patients received optimal medical therapy.

The decision between BA and DES implantation as well as the choice between DES, BA or medical treatment in cases of recurrent restenosis were left to the operator. Patients in the DES group received DAPT for one year, whereas patients in the BA received aspirin only.

Intraobserver and interobserver variabilities of ISR analysis were assessed in a subset of 50 patients. Interpretations of the two investigators on the presence or absence of ISR agreed in 92\% and 95\% respectively. Intraobserver variability was assessed by one investigator. The concordance rate of the two readings for the presence or absence of ISR was $94 \%$ and $95 \%$ respectively.

\section{Statistical analysis}

Continuous variables are expressed as mean \pm SD. Categorical variables are expressed as percentages. To compare parametric continuous variables, Student's t-test was used; to compare nonparametric continuous variables, the Mann Whitney U test was used; and to compare categorical variables, chi-squared test was used. Multivariate logistic regression analysis was carried out to identify the independent predictor of MACE. Event-free survival curves were generated by the 
Table 1

Patients' clinical characteristics.

\begin{tabular}{llll}
\hline & BA & DES & $\mathrm{p}$ \\
\hline Male, n (\%) & $91(79.1 \%)$ & $134(87 \%)$ & 0.08 \\
Age, (years) & $61.6 \pm 11.0$ & $61.7 \pm 10.8$ & 0.967 \\
LVEF (\%) & $64.1 \pm 9.3$ & $66.4 \pm 8.7$ & 0.63 \\
Current smoker, n (\%) & $54(47 \%)$ & $68(44.2 \%)$ & 0.648 \\
Family history of CAD, n (\%) & $40(34.7 \%)$ & $55(35.6 \%)$ & 0.40 \\
Diabetes mellitus, n (\%) & $57(49.6 \%)$ & $64(41.6 \%)$ & 0.192 \\
Hypertension, n (\%) & $65(56.5 \%)$ & $77(50 \%)$ & 0.550 \\
In-hospital medical treatment, n (\%) & & & \\
$\quad$ ACE-I/ARB & $47(41 \%)$ & $77(50 \%)$ & 0.645 \\
Beta blocker & $63(55.1 \%)$ & $91(59.2 \%)$ & 0.26 \\
Calcium channel blocker & $20(17.4 \%)$ & $26(17.1 \%)$ & 0.30 \\
Statins & $64(56 \%)$ & $83(54 \%)$ & 0.13 \\
Fasting glucose, (mg/dL) & $101 \pm 10$ & $89 \pm 11$ & 0.50 \\
HDL-C, (mg/dL) & $42 \pm 13$ & $44 \pm 9.9$ & 0.24 \\
LDL-C, (mg/dL) & $140 \pm 56$ & $135 \pm 28$ & 0.41 \\
Hemoglobin, (g/L) & $13.0 \pm 1.4$ & $13.3 \pm 1.4$ & 0.60 \\
Creatinine, (mg/dL) & $0.89 \pm 0.27$ & $0.94 \pm 0.30$ & 0.08
\end{tabular}

ACE-I; angiotensin converting enzyme inhibitor, ARB; angiotensin receptor blocker, $B A$; balloon angioplasty, CAD; coronary artery disease, DES: drug eluting stent, HDL-C; high density lipoprotein cholesterol, LVEF; left ventricular ejection fraction, LDL-C; low density lipoprotein cholesterol.

Kaplan-Meier method and differences in survival rates were compared using log-rank test. All variables showed significance values less than 0.05 . Two-tailed P values less than 0.05 with a confidence interval of 95\% were considered as significant. All statistical studies were performed by using the SPSS program (version 22.0; SPSS Inc., Chicago, Illinois, USA).

\section{Results}

Baseline clinical and demographic characteristics were similar in both groups. There was no difference between the two groups in terms of age, sex, hypertension, hyperlipidemia, diabetes mellitus, smoking, LVEF and in-hospital medical treatment. Table 1 shows the clinical characteristics of the patients.

Table 2

Patients' angiographic and procedural characteristics.

\begin{tabular}{|c|c|c|c|}
\hline & $\mathrm{BA}$ & DES & $\mathrm{p}$ \\
\hline \multicolumn{4}{|l|}{ Indication for stenting, $\mathrm{n}(\%)$} \\
\hline Stable angina pectoris & $72(47 \%)$ & $91(59 \%)$ & 0.11 \\
\hline Unstable angina pectoris & $33(27 \%)$ & $50(31 \%)$ & 0.23 \\
\hline Myocardial infarction & $10(8.6 \%)$ & $13(10 \%)$ & 0.45 \\
\hline Coronary vessel treated, n (\%) & & & 0.01 \\
\hline LAD & $43(40.6 \%)$ & $83(57.6 \%)$ & \\
\hline LCX & $26(24.5 \%)$ & $22(15.3 \%)$ & \\
\hline RCA & $35(33 \%)$ & $31(21.5 \%)$ & \\
\hline $\begin{array}{l}\text { Number of treated coronary artery, } \\
n \geq 2\end{array}$ & $2(1.9 \%)$ & $8(5.6 \%)$ & \\
\hline Stent length, (mm) & $18.9 \pm 5.9$ & $16.0 \pm 6.6$ & 0.05 \\
\hline Stent diameter, (mm) & $2.70 \pm 0.54$ & $3.32 \pm 0.65$ & 0.04 \\
\hline \multicolumn{3}{|l|}{ Lesion morphology of the previous } & 0.723 \\
\hline procedure, $\mathrm{n}(\%)$ & $15(13 \%)$ & $11(7.1 \%)$ & \\
\hline A & $24(20.8 \%)$ & $37(24.2 \%)$ & \\
\hline B1 & $66(57.4 \%)$ & $94(60.9 \%)$ & \\
\hline B2 & $10(8.6 \%)$ & $12(7.8 \%)$ & \\
\hline \multicolumn{4}{|l|}{$\mathrm{C}$} \\
\hline \multicolumn{3}{|l|}{ In-stent restenosis pattern by Mehran } & $<0.0001$ \\
\hline classification, $\mathrm{n}(\%)$ & $42(40.7 \%)$ & $64(33.1 \%)$ & \\
\hline Pattern I: focal & $46(39.3 \%)$ & $51(32.9 \%)$ & \\
\hline Pattern II: (diffuse, intrastent) & $25(22.1 \%)$ & $20(13 \%)$ & \\
\hline Pattern III: (proliferative) & $0(0 \%)$ & $19(12.3 \%)$ & \\
\hline Pattern IV: (occlusion) & & & \\
\hline
\end{tabular}

$\overline{\text { LAD; left anterior descending artery, RCA; right coronary artery; LCX; left circumflex }}$ artery.
Table 3

1-year follow-up results of the patients.

\begin{tabular}{lllc}
\hline & BA & DES & p \\
\hline MACE, n (\%) & $33(28.7 \%)$ & $13(8.7 \%)$ & $<0.0001$ \\
Death, n (\%) & $15(13 \%)$ & $11(7.1 \%)$ & 0.105 \\
MI, n (\%) & $8(7 \%)$ & $10(6.5 \%)$ & 0.880 \\
Target lesion revascularization, n (\%) & $23(20 \%)$ & $11(7.1 \%)$ & 0.002
\end{tabular}

MACE; major adverse cardiac events, MI; myocardial infarction.

The indications for stenting were similar in both groups. Elapsed time between stent implantation and ISR intervention was $9.8 \pm$ 9.3 months. There were no statistically significant differences between the two groups in terms of lesion type and stent length. Compared with BA group, DES group had more LAD lesions involved and larger stent diameter (Table 2). BA was used predominantly in class I and II lesions, whereas class III and IV lesions were treated with DES. Repeat coronary angiography was performed at $9.3 \pm 8.7$ and $13.8 \pm 10.5$ months $(\mathrm{p}=0.03)$ in BA and DES groups, respectively. Patients in the BA and DES groups were followed-up for $51.05 \pm 12.7$ and $54.5 \pm 13.9$ months $(\mathrm{p}=0.147)$, respectively. Procedural and angiographic variables are shown in Table 2.

At one-year follow-up, patients in the DES group showed significantly lower MACE and TVR. MI and mortality rates were not significantly different between two groups. 1-year follow-up results are given in Table 3.

A mean of 5 years follow-up resulted in a significantly higher composite of MACE including death, MI and TVR, recurrent angina and TVR in BA group than those in the DES group. Cardiac mortality and MI rates were similar in both groups. TVR for recurrent restenosis occurred earlier in the BA group than that in the DES-group. Numbers of repeated target lesion and restenosis rate were both higher for the BA group than for the DES group. The incidence of definite stent thrombosis was similar in both groups. Of all six patients with stent thrombosis, four had stopped antiplatelet therapy prior to elective surgery and only two patients were on DAPT. Five-year follow-up results are given in Table 4.

Fig. 2 shows the 5-year event-free survival of the patients. MACE-free 1-year survival and 5-year survival rates were significantly higher in DES group compared to BA group. There was a progressive decrease in LVEF and renal function in BA group compared to DES group. However, these differences were not statistically significant.

Table 5 shows the results of the multivariate analysis to identify the predictors of MACE. Diabetes, lesion type, treatment device (BA or DES)

Table 4

5-year follow-up results of the patients.

\begin{tabular}{lllc}
\hline & BA & DES & $\mathrm{p}$ \\
\hline Follow-up duration, years & $4.21 \pm 1.03$ & $4.49 \pm 1.13$ & 0.043 \\
Composite MACE, n (\%) & $58(50.4 \%)$ & $49(31.8 \%)$ & 0.002 \\
Cardiac death, n (\%) & $25(21.7 \%)$ & $25(16.2 \%)$ & 0.251 \\
CABG surgery, n (\%) & $5(4.3 \%)$ & $6(3.9 \%)$ & 0.380 \\
MI, n (\%) & $7(6.1 \%)$ & $8(5.2 \%)$ & 0.752 \\
Recurrent angina, n (\%) & $49(42.6 \%)$ & $42(27.3 \%)$ & 0.009 \\
Time to repeat revascularization & $8.7 \pm 4.2$ & $12.5 \pm 4.4$ & 0.02 \\
$\quad$ months, n (\%) & & & \\
Target lesion revascularization, n (\%) & $43(37.4 \%)$ & $32(20.8 \%)$ & 0.003 \\
Repeated target lesion, n & $2.0 \pm 0.8$ & $1.38 \pm 0.5$ & 0.01 \\
Stent thrombosis, n (\%) & $1(0.9 \%)$ & $6(3.9 \%)$ & 0.123 \\
Acute, n (\%) & $1(0.9 \%)$ & $1(0.6 \%)$ & \\
Subacute, n (\%) & $0(0 \%)$ & $1(0.6 \%)$ & \\
Late, n (\%) & $0(0 \%)$ & $4(2.5 \%)$ & \\
New lesion stenting, n (\%) & $8(7 \%)$ & $9(\% 5.8)$ & 0.711 \\
Cardiac event free survival, n (\%) & $57(49.6 \%)$ & $105(68.2 \%)$ & $<0.0001$ \\
Restenosis rate & $44(38.3 \%)$ & $33(21.4 \%)$ & 0.003 \\
\hline
\end{tabular}

CABG surgery; coronary artery bypass graft surgery, MACE; major adverse cardiac events, MI; myocardial infarction. 


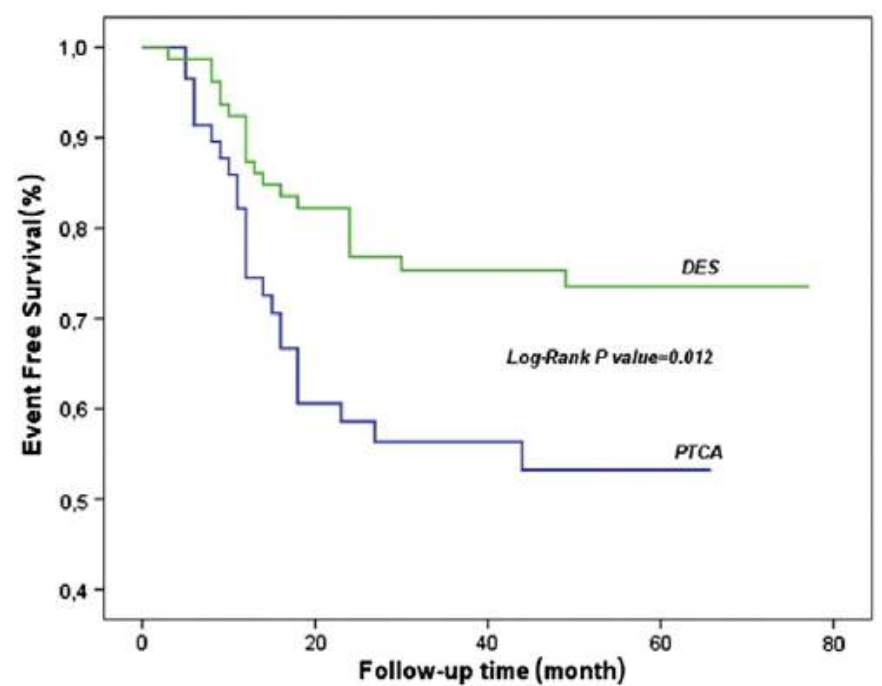

Fig. 2. Kaplan-Meier analysis of the event-free survival at five years according to treatment group.

and Mehran classification were independent predictors of MACE in multivariate analysis.

\section{Discussion}

The main finding of our study was that, at 5-year follow-up, composite MACE, recurrent angina and TVR were significantly lower and MACEfree survival was significantly better in the DES treated patients. However mortality and risk of MI did not differ between two groups. DES use was associated with lower rates of composite MACE and TVR, although it was utilized in patients with more complicated lesions.

Coronary stent implantation is frequently performed in percutanous coronary interventions. ISR is a major late complication following BMS implantation, which occurs in $10-80 \%$ of lesions treated in daily practice. ${ }^{8}$ BMS-ISR is an independent predictor of mortality. ${ }^{15,16}$ The use of BMS is associated with high restenosis rate (up to 25\%). Although DES dramatically reduced the rate of restenosis to less than $10 \%,{ }^{17,18}$ it is associated with increased risk for late stent thrombosis and requires prolonged DAPT. $^{19}$

Any percutaneous coronary intervention causes trauma to the vessel wall. ISR is generally viewed as a healing response after injury incurred during stent placement. This is characterized by a sequence of inflammation, granulation, extracellular matrix remodeling and smooth muscle cell proliferation. These processes lead to neointimal hyperplasia. Predictive factors of restenosis can be classified into patient-related, lesion-related and procedure-related factors. Lesion-related factors can be listed as vessel diameter, tortuous vessels, calcified lesions and

Table 5

Multivariate predictors of composite MACE.

\begin{tabular}{llcl}
\hline & OR & $\% 95$ CI & p \\
\hline HT & 1.24 & $0.73-2.11$ & 0.417 \\
DM & 0.466 & $0.291-0.843$ & 0.003 \\
Number of diseased coronary arteries & 1.13 & $0.69-1.84$ & 0.624 \\
Lesion type & 0.66 & & 0.001 \\
Stent length (mm) & 0.993 & $0.948-1.04$ & 0.779 \\
Mehran classification & 2.82 & $2.09-3.89$ & 0.000 \\
Number of stents used & 0.789 & $0.505-1.23$ & 0.300 \\
Stent diameter & 0.684 & $0.312-1.49$ & 0.341 \\
Treatment with BA or DES & 2.18 & $1.32-3.59$ & 0.002 \\
\hline
\end{tabular}

BA, balloon angioplasty, CI; confidence interval, DES; drug eluting stent, DM; diabetes mellitus, HT; hypertension, OR; odds ratio. previous restenosis. Patient-related factors include patient age, diabetes mellitus and genetic factors. Procedural characteristics predicting ISR are residual dissection and length of stented segment. ${ }^{20}$

Several therapeutic options to treat ISR have been proposed, such as repeat BA, repeat stenting, cutting balloon angioplasty, directional coronary atherectomy, rotational coronary atherectomy, brachytherapy, DES, molecular biology and genetics. BA has been used frequently, as it is relatively inexpensive and easy to perform. DES have achieved great success in treating patients with de-novo lesions. These findings provide a hope for the treatment of ISR. Sirolimus and paclitaxel inhibit smooth muscle cell proliferation and migration in vitro and in vivo. ${ }^{21,22}$ Several studies have demonstrated that stents eluting sirolimus (Cypher, Cordis a Johnson and Johnson Company) and paclitaxel (TAXUS NIRx, Boston Scientific Corporation) reduce ISR. ${ }^{23-27}$ It has been shown that oral therapy with sirolimus before and after repeat intervention results in a significant improvement in the angiographic parameters of restenosis. ${ }^{28}$ Paclitaxel-coated balloon catheters reduce repeat restenosis in patients with ISR. ${ }^{29}$

In spite of insufficient data, interventional cardiologists commonly use the stent sandwich technique to treat ISR. Final lumen crosssectional area is the independent predictor of subsequent ISR and TVR. Full stent expansion with sufficient lumen area (lumen area $90 \%$ or greater of the average reference lumen area preintervention) minimizes restenosis. Compared to BA, coronary stenting achieved better initial angiographic results but fails to improve restenosis rate in patients with ISR. However, patients with large vessels ( $\geq 3 \mathrm{~mm}$ ) have a better outcome after repeat stenting. ${ }^{4}$

ISAR-DESIRE study randomized 300 patients with BMS ISR to treatment with SES, PES and BA. ${ }^{30}$ At 1-year follow-up, the use of DES led to significantly lower rates of TVR. DES were markedly superior to conventional balloon angioplasty for the treatment of BMS-ISR. RIBS-II compares the results of SES with those of BMS in patients with ISR. ${ }^{11}$ At 1 year, the event-free survival was better in the SES group. At 4 years, the event-free survival was $76 \%$ in the SES arm and $65 \%$ in the $\mathrm{BA}$ arm $(\mathrm{p}=0.019)$. Same researchers published the results of $450 \mathrm{pa}-$ tients with ISR treated with BA or repeat BMS implantation. ${ }^{4}$ In that study, binary restenosis rate, TVR and one-year event-free survival were similar in both groups.

In our study, MACE and TVR rates were consistent with other studies. Myocardial and cardiac deaths were similar in both groups. During follow-up MACE rates were significantly lower and MACE-free survival was significantly better in the DES treated patients.

\section{Conclusions}

ISR has been a longstanding problem after percutaneous coronary intervention. The pathophysiology of ISR is multifactorial. There are several options for therapy and treatments that should therefore be individualized. Although DES have significantly reduced in-hospital complications, ISR and TVR, BA compared with DES resulted in similar rates of mortality and MI. Hence, BA may be the preferred method of treatment. Recent advances in DES technology have allowed cardiologists to treat much more complicated lesions and have dramatically reduced the rates of ISR and TVR.

\section{Study limitations}

Our study has several limitations. It was a retrospective analysis of data at a single center and the number of patients was small. Discontinuation of medical treatment during follow-up may affect the patient outcomes. Decision between BA and DES implantation was left up to the operator. Another limitation was that the current funding restrictions in our country affected the operator preferences and resulted in lower DES use. 


\section{Declaration of conflicting interests}

The author(s) declared no potential conflicts of interest with respect other research, authorship, and/or publication of this article.

\section{Funding}

The author(s) received no financial support for the research, authorship, and/or publication of this article.

\section{Conflict of interest}

None declared.

\section{References}

1. Cutlip DE, Chauhan MS, Baim DS, et al. Clinical restenosis after coronary stenting: perspectives from multicenter clinical trials. J Am Coll Cardiol 2002;40(12):2082.

2. Díaz JF, de La Torre JM, Sabaté M, Goicolea J. Spanish Cardiac Catheterization and Coronary Intervention Registry. 21st official report of the Spanish Society of Cardiology Working Group on Cardiac Catheterization and Interventional Cardiology (19902011), Vol. 65 (12). Rev Esp Cardiol (Engl Ed); 2012. p. 1106-1116.

3. Pellegrini DO, Gomes VO, Lasevitch R, et al. Efficacy and safety of drug-eluting stents in the real world: 8-year follow-up. Arq Bras Cardiol 2014 Sep;103(3):174-182.

4. Alfonso F, Zueco J, Cequier A, et al. A randomized comparison of repeat stenting with balloon angioplasty in patients with restenosis after coronary stenting. $\mathrm{J}$ Am Coll Cardiol 2003;42(5):796-805.

5. Kastrati A, Mehilli J, von Beckerath N, et al. Sirolimus-eluting stent or paclitaxel-eluting stent vs balloon angioplasty for prevention of recurrences in patients with coronary in-stent restenosis: a randomized controlled trial. JAMA 2005;293(2):165-171

6. Alfonso F. Treatment of drug-eluting stent restenosis the new pilgrimage: quo vadis. $J$ Am Coll Cardiol 2010;55(24) [2717-2120].

7. Weintraub WS, Ghazzal ZM, Douglas Jr JS, et al. Long-term clinical follow-up in patients with angiographic restudy after successful angioplasty. Circulation 1993;87(3):831-840.

8. Mehran R, Dangas G, Abizaid AS, et al. Angiographic patterns of instent restenosis. Classification and implications for long-term outcome. Circulation 1999;100(18): 1872-1878.

9. Alfonso F, Cequier A, Angel J, et al. Value of the American College of Cardiology/American Heart Association angiographic classification of coronary lesion morphology in patients with in-stent restenosis. Am Heart J 2006;151(3): 681.e1-681.e9.

10. Al Muradi H, Mehra A, Okolo J, et al. Clinical presentation and predictors of target vessel revascularization after drug-eluting stent implantation. Cardiovasc Revasc Med 2012;13(6):311-315.

11. Alfonso F, Pérez-Vizcayno MJ, et al. A randomized comparison of sirolimus-eluting stent with balloon angioplasty in patients with in-stent restenosis: results of the Restenosis Intrastent: Balloon Angioplasty Versus Elective Sirolimus-Eluting Stenting (RIBS-II) trial. J Am Coll Cardiol 2006:47(11):2152-2160.

12. Cutlip DE, Windecker S, Mehran R, et al. Academic Research Consortium. Clinical end-points in coronary stent trials. A case for standardized definitions. Circulation 2007;115(17):2344-2351.

13. Smith Jr SC, Feldman TE, Hirshfeld Jr JW, et al. ACC/AHA/SCAI 2005 guideline update for percutaneous coronary intervention summary article: a report of the American
College of Cardiology/American Heart Association Task Force on Practice Guidelines (ACC/AHA/SCAI Writing Committee to Update the 2001 Guidelines for Percutaneous Coronary Intervention). Circulation 2006;113(1):156-175.

14. Nebeker JR, Virmani R, Bennett CL, et al. Hypersensitivity cases associated with drug-eluting coronary stents: a review of available cases from the Research on Adverse Drug Eventsand Reports (RADAR) project. J Am Coll Cardiol 2006;47(1): $175-181$.

15. Schühlen $\mathrm{H}$, Kastrati A, Mehilli J, et al. Restenosis detected by routine angiographic follow-up and late mortality after coronary stent placement. Am Heart J 2004;147(2):317-322.

16. Chen YL, Chen MC, Wu CJ, et al. Impact of 6-month angiographi restenosis inside bare-metal stents on long term clinical outcome in patients with coronary artery disease. Int Heart J 2007;48(4):443-454.

17. Moses JW, Leon MB, Popma JJ, et al. Sirolimus-eluting stents versus standard stents in patients with stenosis in a native coronary artery. N Engl J Med 2003;349(14): 1315-1323.

18. Stone GW, Ellis SG, Cox DA, et al. A polymer-based, paclitax eleluting stent in patients with coronary artery disease. N Engl J Med 2004;350(3):221-231.

19. Kalesan B, Pilgrim T, Heinimann K, et al. Comparison of drug-eluting stents with bare metal stents in patients with ST-segment elevation myocardial infarction. Eur Heart J 2012;33(8):977-987.

20. Bhatia Vineet, Kaul Upendra. Management of in-stent restenosis after DES use. Card Interv Today 2011(July/August):50-55.

21. Marx SO, Jayaraman T, Go LO, Marks AR. Rapamycin-FKBP inhibits cell cycle regulators of proliferation in vascular smooth muscle cells. Circ Res 1995;76(3): 412-417.

22. Poon M, Marx SO, Gallo R, Badimon JJ, Taubman MB, Marks AR. Rapamycin inhibits vascular smooth muscle cell migration. J Clin Invest 1996;98(10):2277-2283.

23. Sousa JE, Costa MA, Abizaid AC, et al. Sustained suppression of neointimal proliferation by sirolimus-eluting stents: one-year angiographic and intravascular ultrasound follow-up. Circulation 2001;104(17):2007-2011.

24. Degertekin M, Serruys PW, Foley DP, et al. Persistent inhibition of neointimal hyperplasia after sirolimus-eluting stent implantation: long-term (up to 2 years) clinical, angiographic, and intravascular ultrasound follow-up. Circulation 2002;106(13): 1610-1613.

25. Morice MC, Serruys PW, Sousa JE, et al. Randomized study with the sirolimus-coated Bx velocity balloon-expandable stent in the treatment of patients with de novo native coronary artery lesions. A randomized comparison of a sirolimus-eluting stent with a standard stent for coronary revascularization. N Engl J Med 2002;346(23): 1773-1780.

26. Grube E, Silber S, Hauptmann KE, et al. TAXUS I: six- and twelve-month results from a randomized, double-blind trial on a slowrelease paclitaxel-eluting stent for de novo coronary lesions. Circulation 2003;107(1):38-42.

27. Colombo A, Drzewiecki J, Banning A, et al. TAXUS II study group. randomized study to assess the effectiveness of slow- and moderate-release polymerbased paclitaxel-eluting stents for coronary artery lesions. Circulation 2003;108(7): 788-794.

28. Hausleiter J, Kastrati A, Mehilli J, et al. Randomized, double- blind, placebo-controlled trial of oral sirolimus for restenosis prevention in patients with in-stent restenosis: the oral sirolimus to inhibit recurrent in-stent stenosis (OSIRIS) trial. Circulation 2004;110(7):790-795.

29. Scheller B, Hehrlein C, Bocksch W, et al. Treatment of coronary in-stent restenosis with a paclitaxel-coated balloon catheter. N Engl J Med 2006;355(20):2113-2124.

30. Kastrati A, Mehilli J, von Beckerath $\mathrm{N}$, et al. ISAR-DESIRE study investigators. Sirolimus-eluting stent or paclitaxel-eluting stent vs balloon angioplasty for prevention of recurrences in patients with coronary in-stent restenosis: a randomized controlled trial. JAMA 2005;293(2):165-171. 